
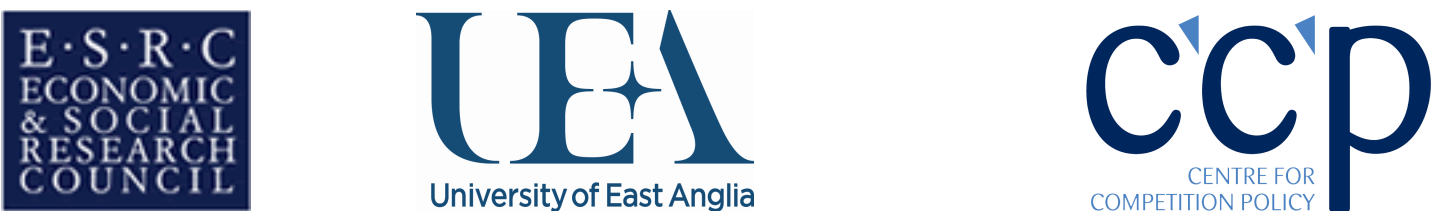

\title{
Collusion and Strategic Favoritism in Organizations
}

\author{
by \\ Zhijun Chen \\ ESRC Centre for Competition Policy, University of East Anglia and \\ School of Economics, Zhejiang University
}

CCP Working Paper 08-27

The support of the Economic and Social Research Council is gratefully acknowledged. 


\title{
Collusion and Strategic Favoritism in Organizations
}

\author{
Zhijun Chen ${ }^{1}$ \\ The ESRC Centre for Competition Policy, University of East Anglia and the \\ College of Economics, Zhejiang University \\ E-mail: chenzj1219@gmail.com
}

Version: August, 2008

\begin{abstract}
Fighting collusion has long been a challenge in organizations, whilst favoritism in organizations has long been attacked as one of the most important sources of workplace conflicts. This paper links the phenomena of collusion and favoritism together which seem to be irrelevant. We show that favoritism cannot benefit organizations where collusion is not a serious concern, meanwhile explicit favoritism is not effective in dealing with wellorganized collusion; however strategic use of favoritism can bring conflicts among collusive subordinates and undermine the efficiency of collusion, therefore it is effective in fighting collusion.
\end{abstract}

JEL Classification: C72, D82

Key Words: Collusion, Favoritism, Tournaments

\section{INTRODUCTION}

Objective measures of employee performance are rarely available in organizations like firms and bureaucracies. Instead these organizations rely on subjective assessments by supervisors, which may cause serious incentive problems. The employers have incentives to underestimate employee performance and renege on payments, which will mitigate employee incentives in working.

Rank-order tournaments are widely adopted to resolve these incentive problems. By granting a reward only to the winner of the contest, tournaments trigger a competition between employees and thus provide strong incentives to exert high efforts; meanwhile the fixed amount of prize plays a role of commitment which overcomes the supervisor's temptation of renege.

Economists have long emphasized the importance and effectiveness of tournaments, ${ }^{2}$ however, economists rarely address the fact that tournaments are vulnerable to collusion or collective sabotages. ${ }^{3}$ Since the rank order of performance is determined by employees' relative rather than absolute effort levels, the order will remain

\footnotetext{
${ }^{1}$ This paper is revised from a chapter of my Ph.d thesis in Toulouse School of Economics. I am grateful to my supervisor Patrick Rey for his valuable guidance and encouragements. I am also grateful to Jacques Cremer, Morten Hviid, Shingo Ishiguro, Hideshi Itoh, Bruno Jullien, David Martimort and Jean Tirole for their important comments, as well as seminar participants in Toulouse and in the Contract Theory Workshop in Osaka University. Financial support from the Economic and Social Research Council (ESRC) of UK is greatly acknowledged.

${ }^{2}$ For instance, see Green and Stokey (1983), Lazear and Rosen (1981), Nalebuff and Stiglitz (1983).

${ }^{3}$ Economists have paid much attention to the fact of individual or noncooperative sabotages in tournaments, where employees expend some resources in undercutting the performance of their rivals; see Kong-Pin Chen (2003) for instance.
} 
unchanged if all employees cut their efforts collectively by same levels; this brings each employee the same expected reward as before but saves his effort cost.

The phenomenon of collusion is indeed quite common in organizations. ${ }^{4}$ Miller (1992) describes a so-called "binging" game played between workers when discussing the compensation scheme of the bank wiring room in the Hawthorne plant of Western Electric; this game is played to punish the workers who produce too much, which is indeed a collusion enforcement device to prevent the workers from exerting high effort levels. Perhaps, the most familiar case to economists is the "bidding ring" which prevails not only in procurements but also in R\&D contests. As collusive behaviors are always conducted in secret, what we have observed is only the tip of the iceberg.

On the other hand, subjective assessments of employee performance open the door to favoritism, where employers act on personal preference toward subordinates to favor some employees over others. Favoritism becomes one of the most important sources of conflicts in organizations, ${ }^{5}$ and its harmful effects on the design of incentives are widely discussed in the existing literature. ${ }^{6}$ However, Prendergast and Topel (1996) find that favoritism can generate value for those who exercise it. In their analysis, those in authority - who are called supervisors - derive utility form exercising bias, which leads naturally to a demand for "power" by those in authority; this demand can mean that favoritism benefits the organization. In their model, those in authority value their power to affect subordinates' welfare because their utilities are derived directly from the subordinates' payoff, and therefore they address the phenomenon of favoritism in organizations ad hoc.

While ad hoc favoritism in organizations is an interesting case, it is far from a general case as there is little evidence that shows that bringing individual preference towards some subordinate into incentive schemes can improve the employer welfare directly. ${ }^{7}$ We are interested in more general cases such as when favoritism is used strategically in organizations where the employer welfare does not depend on personal preference over employees.

This paper bridges the phenomenon of strategic favoritism in organizations to the mission of fighting collusion; we find that the strategical use of favoritism is an effective measure to fight collusion. Whenever an employer has personal preference toward some subordinate, he may use favoritism to benefit the organization. In particular when the superior finds the subordinates are engaged in collective sabotage, using favoritism strategically can bring conflicts between collusive subordinates and contribute to destabilizing collusion. If the superior uses a biased promotion rule in a tournament which favors his subordinate in the assessment of performance, the favored subordinate is more likely to get the reward even when he takes the same effort as others; therefore more stakes of collusion should be allocated to at-

\footnotetext{
${ }^{4}$ See Tirole $(1986,1992)$ for detailed discussion.

${ }^{5}$ For instance, Comerford (2002) finds that favoritism is the second most important source of workplace conflict followed by workload, in a survey of Canadian government workers.

${ }^{6}$ For instance, Prendergast and Topel (1993) investigate the phenomena of discretion and bias in performance evaluation in organizations, and they argue that favoritism can give rise to inefficiency on two margins. The first is rent seeking by workers, which is usually a waste of time. The second occurs because bias makes it difficult to determine the true talents of workers. Both were used to explain a tendency towards equality in organizations.

${ }^{7} \mathrm{Kim}, \mathrm{Qin}$ and $\mathrm{Yu}(2006)$ investigates the cases where a supervisor can take bribes from subordinates; they study how the bribery game affects the supervisor's optimal design of a rank-order tournament and how the anticipation of the bribery game affects the agents' optimal effort choices. We think corruption is a serious problem in organizations, but it is not a general case since taking bribes is against the law.
} 
tract his participation into collusive activities. So doing will shrink the stake of the unfavored employee and cause a conflict in the allocation of collusive stakes, however the dispute in stake allocation can be easily settled when the identity of the favored subordinate is commonly known, through a mechanism of side payments which leaves the favored one more stakes according to his status quo utility.

Whenever the employer reveals his preference only to one employee, say an informed subordinate, ${ }^{8}$ the identity of the favorite becomes a piece of private information. Strategic revelation of information brings asymmetric information among collusive subordinates; and overcoming the incentive problem under asymmetric information leads to a distortion of efficiency which contributes to destabilizing collusion.

The strategic use of favoritism to fight collusion highlights the spirit of "divide and conquer"; it also implies that the relationships between the supervisor and subordinates in organizations are far more complicated than what we have observed, as most activities like collusion and strategic favoritism are conducted in secret. Prendergast and Topel (1996) point out that "Economists rarely address the fact that firms are social institutions, where personal relations among coworkers, bosses, and subordinates constitute an important component of many workers' daily lives" (P. 958). Personal relations among subordinates are built mainly for coordination but they also play an important role in facilitating collusion, meanwhile subordinates often invest many resources in the relationship with the superior to seek favoritism in promotion. These relationships act as informal mechanisms which affect the effectiveness of incentive schemes, and they can be also utilized strategically to benefit organizations. ${ }^{9}$ However, observations of these informal tricks of governance like strategic favoritism are quite few since no superior wants to show off these skills. ${ }^{10}$

Ishiguro (2004) is, to our knowledge, the first paper that investigates the role of favoritism in fighting collusion under tournaments. He shows that inducing socially efficient effort levels is impossible when collusion is accomplished by an enforceable contract; moreover a discriminatory mechanism that offers incentive schemes based on employee identities (i.e. favored or unfavored) can induce the favored subordinate (and only the favored one) to exert the high effort, given that the ranking of relative performance is verifiable. However, the incentive scheme he proposed involves a naked discrimination based on identities which are exogenously given, such as sex or race, thus cannot be applied in general cases as naked discrimination is prohibited by law. Moreover, while there are some cases that the ranking of relative performance is verifiable such as football matches, they are far from general cases in organizations where objective measures of performance are rarely available.

Our paper focuses on fighting collusion in a generalized tournament mechanism where only subjective assessments of employee performance are available, and analyzes the role of implicit discrimination or favoritism where the employer can

\footnotetext{
${ }^{8}$ For instance, the informed employee may be a neighbor of the employer who has a close relationship with the superior.

${ }^{9}$ Personal relations in organizations are widely discussed in traditional organizational theory based in psychology, sociology, and political science. Gary Miller (1992) emphasizes that social interactions including gambling, games, and afterwork activities that are not directly related to work, serve important purpose; they provide a means by which the members of the social cliques could demonstrate to one another in a continuous way their trustworthiness and capacity to cooperate (see chapter 9).

${ }^{10}$ These skills are often regarded as immoral tricks against employees which bring conflicts and stresses in organizations.
} 
impose some bias in the assessment of performance. ${ }^{11}$ Moreover, we show that the efficiency of collusion can be mitigated whenever the employer uses favoritism strategically to introduce asymmetric information among collusive employees, in which case inducing socially efficient efforts is possible.

Our analysis also contributes to the literature of favoritism in organizations. Prendergast and Topel (1996) bring an excellent explanation of how favoritism generates value for organizations in a model where those in authority value their power to affect subordinates' welfare and their demands for this power are traded off against the costs of favoritism caused by added arbitrariness of performance evaluations. Meanwhile Kwon (2006) argues that favoritism can arise endogenously as an optimal decision rule in a model of strategic delegation of decisions where two experts produce competing ideas with conflicts in preference. Our paper shows that favoritism cannot benefit organizations absent collusion and explicit favoritism is not effective in dealing with well-organized collusion; however strategic use of favoritism can bring conflicts among collusive subordinates and undermine the efficiency of collusion, and it is therefore an effective measure in fighting collusion.

This paper is also related to the literature of fighting collusion in the environments where the employee performance is verifiable and thus contractible. For instance, Varian (1990) shows that preventing collusion is costly to the principal if agents' actions are privately observable. Whenever the agents' actions are mutually observable, however, Itoh (1993) shows that the principal can benefit from agents' side contracting and mutual monitoring, but the first-best outcome cannot be achieved due to perfect collusion.

Our research also contributes to the literature of collusion-proof mechanism design that has developed rapidly since the seminal paper by Tirole (1986). In the framework of adverse selection, Laffont and Martimort (1997) have shown that collusion-proof contracts can be implemented without any additional costs when the agents' types are uncorrelated; whereas preventing collusion entails additional costs whenever the agents' types are correlated as shown by Laffont and Martimort (2000). Che and Kim (2005) develop a general method for collusion-proof mechanism design, which utilizes the idea of "selling the firm to the agents". They show that any payoff attainable by the principal in the absence of collusion can be attained in the presence of collusion, in a large class of environments with risk-neutral agents for both uncorrelated and correlated types cases whenever there are more than two agents.

The rest of the paper is organized as follows. We present a simple tournament model in section 2 and then derive the optimal tournament contract in the absence of collusion in section 3 as a benchmark. We show in section 4 that the optimal tournament mechanism is vulnerable to collusion and it is impossible to implement the socially efficient efforts when the collusive contract is enforceable. We discuss strategic favoritism in section 5 and show that implementing the efficient effort levels is possible whenever the employer uses favoritism strategically. Finally, we conclude in section 6 .

\footnotetext{
${ }^{11}$ The employer's bias in the assessment of employee performance is indeed quite common in organizations.
} 


\section{THE MODEL}

The model has one principal and two identical agents, $A_{1}$ and $A_{2}$, who are recruited from a competitive labor market with reservation salary 0 . The principal and agents are risk-neutral; in addition the agents are protected by limited liability. Each agent $i, i=1,2$, can choose an effort $e^{i} \in\{1,0\}$, namely "work" or "shirk", which is unobservable by others. The effort cost is denoted by $C\left(e^{i}\right)$ and it is assumed that only the high effort incurs a positive labor cost $C(1)=c>0$.

The production process of each project is independent and the output of agent $A_{i}$ is given by $y^{i}=e^{i}+\varepsilon^{i}$, where $\varepsilon^{i}$ represents a random shock. The random variables $\varepsilon^{i}, i=1,2$, are identically and independently distributed with a symmetric distribution function $F($.$) on \mathbb{R}$, where $F($.$) is twice-differentiable and has symmetric$ properties: $F(0)=1 / 2$ and $F(x)=1-F(-x)$ for any $x \in \mathbb{R}$. The corresponding density function, as denoted by $f($.$) , satisfies f(x)=f(-x)$ for any $x \in \mathbb{R}$; in addition it is assumed to be strictly increasing for $x \leq 0$ and strictly decreasing for $x \geq 0 .{ }^{12}$

The objective assessments of agent performance $y_{i}$ are not available and any incentive contracts contingent on unverifiable performance are thus incomplete. ${ }^{13}$ The performance of each agent can be related in spite of the independence of the production process, and therefore the principal can introduce a tournament mechanism with a fixed prize $T$ for the winner (and only the winner) of the contest. ${ }^{14}$ The winner will be selected based on a promotion rule which is announced at the beginning of the game.

A promotion rule $H$ specifies conditions under which an agent will win in the tournament. For instance, an unbiased rule specifies that the agent $A_{i}$ wins the prize if and only if his performance is better than his peer, that is $y^{i}>y^{j}$. However, subject assessments of employee performance open the door to favoritism which allows the principal to favor some agent in the evaluation of performance and bring a bias in promotion. Suppose the principal prefers the agent $A_{i}$; she can overestimate the performance of the agent $A_{i}$ from $y^{i}$ to $y^{i}+b$, where $b$ represents the bias of evaluation, in which case the agent $A_{i}$ wins only if $y^{i}+b>y^{j} .^{15}$

As the probability distribution function of $y^{i}$ is given by $P_{r}\left\{y^{i} \leq y\right\}=F\left(y-e^{i}\right)$, the distribution function of $\varepsilon^{i}-\varepsilon^{j}$ can be derived by

$$
G(x) \equiv P_{r}\left\{\varepsilon^{i}-\varepsilon^{j} \leq x\right\}=\int_{-\infty}^{+\infty} F(x+\varepsilon) f(\varepsilon) d \varepsilon
$$

Note that $G($.$) also has a symmetric property as G(x)=1-G(-x)$, which follows from

$$
\begin{aligned}
1-G(-x) & =P_{r}\left\{\varepsilon^{i}-\varepsilon^{j} \geq-x\right\} \\
& =P_{r}\left\{\varepsilon^{j}-\varepsilon^{i}<x\right\}=G(x) .
\end{aligned}
$$

\footnotetext{
${ }^{12}$ The basic model is exactly the same as Ishiguro (2004), this makes it simple to compare our results with that paper; it is also one of the standard models in the literature of tournaments.

${ }^{13}$ Ishiguro (2004) assumes in his model that the absolute performance is unverifiable but the relative performance is verifiable; this assumption is restrictive given the nature of subjectivity in performance assessments.

${ }^{14}$ The agent can also get a basic wage whether he wins or loses, which is normalized to zero for simplicity.

${ }^{15}$ Prendergast and Topel (1996) also investigate the phenomonon of favoritism in organizations where supervisors often impose some bias in their evaluations of workers' performance according to their own preferences.
} 
Therefore, the probability that $y^{i}>y^{j}$ happens is given by

$$
P_{r}\left\{y^{j}<y^{i}\right\}=P_{r}\left\{\varepsilon^{j}-\varepsilon^{i} \leq e^{i}-e^{j}\right\}=G\left(e^{i}-e^{j}\right) .
$$

Given an effort pair $e=\left(e^{1}, e^{2}\right)$, the promotion rule $H(b)$ that grants agent $A_{i}$ a bias $b$ in performance assessment induces a probability of winning for the favored agent $A_{i}$ as denoted by $p_{i}(e ; b)$ :

$$
\begin{aligned}
p_{i}(e ; b) & =P_{r}\left\{y^{j}<y^{i}+b\right\}=P_{r}\left\{\varepsilon^{j}-\varepsilon^{i} \leq\left(e^{i}+b\right)-e^{j}\right\} \\
& =G\left(e^{i}+b-e^{j}\right),
\end{aligned}
$$

as well as a winning probability for the unfavored agent $A_{j}$

$$
p_{j}(e ; b)=P_{r}\left\{y^{i}+b<y^{j}\right\}=G\left(e^{j}-e^{i}-b\right)=1-p_{i}(e ; b) .
$$

Under promotion rule $H(b)$, the favored agent is more likely to win than his peer given that both of them exert the same efforts: $p_{i}(e ; b)>p_{j}(e ; b)$.

The simple tournament mechanism which comprises a fixed prize $T$ and a promotion rule $H(b)$ brings the following expected payoffs to agents:

$$
\begin{aligned}
U_{i}(e) & =p_{i}(e ; b) T-C\left(e^{i}\right), \\
U_{j}(e) & =p_{j}(e ; b) T-C\left(e^{j}\right) ;
\end{aligned}
$$

and an expected profit for the principal:

$$
V(e)=E\left[y^{i}+y^{j}\right]-T .
$$

Promotion rules based on relative performance are also unverifiable, but the principal is able to commit a given promotion rule because any deviation from the announced rule cannot increase the principal's profit given that the prize $T$ is fixed. Therefore, favoritism can change the agent payoff but cannot improve the principal's welfare directly; and this is quite different from the setting of Prendergast and Topel (1996) which assumes the supervisor's utility depends on the pay of his subordinate.

\section{TOURNAMENTS ABSENT COLLUSION: A BENCHMARK}

We are interested in how the socially efficient effort levels $\bar{e} \equiv(1,1)$ can be implemented at a minimal incentive cost. As a benchmark, we analyze the simple tournament mechanism in the absence of collusion, with its timing of the game illustrated as follows:

Stage 1: The principal proposes a simple tournament mechanism;

Stage 2: Each agent decides to accept or not; if no one rejects then:

Stage 3: Each agent chooses an effort level;

Stage 4: Outcomes are realized and the prize is awarded to the winner.

The principal aims to induce the efficient effort pair $\bar{e}$ at a minimal prize, therefore the optimal prize $T^{*}$ is the solution of the following optimization program:

(P.1) Min T

Subject to:

$$
\begin{array}{ll}
\left(I R_{i}\right) \quad: & U_{i}(\bar{e})=p_{i}(\bar{e} ; b) T-c \geq 0, \quad i=1,2 ; \\
\left(I C_{i}\right) & : \quad U_{i}(\bar{e}) \geq p_{i}(\widetilde{e} ; b) T, \quad i=1,2
\end{array}
$$


where $\widetilde{e} \equiv(0,1)$ stands for the effort pair whenever the agent $A_{i}$ deviates unilaterally from the efficient effort pair $\bar{e}$.

The agent $A_{i}$ will exert high effort only if the expected payoff is non-negative, as illustrated by his participation constraint $\left(I R_{i}\right)$. Moreover, the tournament mechanism must ensure that he can benefit from working rather than shirking unilaterally, as illustrated by his incentive compatibility constraint $\left(I C_{i}\right)$, which further implies

$$
\left[p_{i}(\bar{e} ; b)-p_{i}(\widetilde{e} ; b)\right] T \geq c .
$$

The following proposition shows that the efficient effort levels can be induced by any simple tournament mechanisms with sufficiently high prize, however the optimal tournament mechanism involves an unbiased promotion rule.

Proposition 1. Absent collusion, a simple tournament mechanism can induce the socially efficient effort levels given that the prize for the winner is sufficiently high; moreover the optimal promotion rule is unbiased.

Proof. Given any promotion rule $H(b)$, the incentive compatibility constraints imply that the prize should be sufficiently high in order to induce agents to take high efforts:

$$
T \geq \max \left\{\frac{c}{p_{1}(\bar{e} ; F)-p_{1}(\widetilde{e} ; F)}, \frac{c}{p_{2}(\bar{e} ; F)-p_{2}(\widetilde{e} ; F)}\right\} .
$$

The optimal prize is thus given by

$$
T^{*}=\frac{c}{G(b)-G(b-1)}=\frac{c}{G(-b)-G(-b-1)},
$$

which implies $G(b)-G(-b)=G(1+b)-G(1-b)$. Note that $G(b)-G(-b)=2 b G^{\prime}(\zeta)$ for some $\zeta$ close to 0 and $G(1+b)-G(1-b)=2 b G^{\prime}(\eta)$ for some $\eta$ close to 1 , therefore $2 b G^{\prime}(\zeta)=2 b G^{\prime}(\eta)$ implies $b=0$ since $G^{\prime}(x)$ is strictly decreasing for $x \geq 0$ and $G^{\prime}(x)=G^{\prime}(-x)$. This shows the optimal tournament mechanism involves an unbiased rule and the optimal prize is given by

$$
T^{*}=\frac{c}{G(1)-G(0)}
$$

A high prize for the winner provides strong incentives for taking high efforts, and the socially efficient effort levels can be implemented with the minimal incentive $\operatorname{cost} T^{*}$ which ensures that taking high efforts forms a Nash equilibrium for both agents. Introducing a bias in performance assessments will benefit the favored agent and provide stronger incentives to work, but this will on the other hand mitigate the incentive of the unfavored one which increases the incentive cost for inducing his high effort. The optimal trade-off of incentive schemes leads to an unbiased promotion rule.

The first result in the proposition is indeed quite common and widely discussed in the literature of tournaments; ${ }^{16}$ while the second one, which shows that favoritism will not benefit organization where collusion is not a concern, is rarely discussed in the existing literature on favoritism. Prendergast and Topel (1996) investigate the cases where the employer will use a supervisor to evaluate the subordinates'

\footnotetext{
${ }^{16}$ See Prendergast (1999) for an excellent survey.
} 
performance and show that favoritism can benefit organizations whenever those in authority value their power to affect subordinates' welfare because their utility are derived directly from subordinates' payoff. Meanwhile in organizations where the employers' welfare does not depends on their preference toward subordinates, which is the case of general interests, our analysis shows that favoritism will not benefit these organizations whenever collusion among subordinates is not a serious problem.

\section{COLLUSION AND EXPLICIT FAVORITISM: AN IMPOSSIBILITY THEOREM}

The simple tournament mechanisms are vulnerable to collusion. The winning probability of each agent is determined by the relative rather than absolute effort levels of agents, and it remains unchanged when both agents cut their efforts coordinately from $\bar{e}$ to $\underline{e} \equiv(0,0)$. But each agent can benefit from saving the effort cost; this provides incentives for agents to collude on low effort levels.

The phenomenon of collusion is quite common in organizations, and fighting collusion has long been a challenge as argued by Tirole (1992):

"Concurrently, sociologists and organization theorists have emphasized that behavior is often best predicted by the analysis of group as well as individual incentives. It would be naive to build incentives for individual members of an organization without considering their effect on collective behavior. In other words, incentive structures must account for the possibility that members collude to manipulate their functioning."

The enforcement of collusive agreements plays a key role to the robustness of collusion; it relies on non-judicial mechanisms in general. Collusive agreements may be enforced by a mechanism of reputation, either by social norms in short-term relationships or by reciprocity in long-term relationships. Miller (1992) investigates a famous case of collective sabotage in the bank wiring observation room at the Hawthorne Works of Western Electric Company, and finds that collusion among workers is enforced by social norms. In the bank wiring room there were two inspectors, nine wiremen, and three solderers. Their task was to connect wires to banks of terminals used in central office telephone switching systems. The fourteen men in the room were paid on a group piecework basis, and each worker was paid based on his relative contribution to the group outputs. The incentive system that was intended to motivate individual contributions was, however, almost completely negated by social norms dictating roughly equal reported output with averaged out to 825 connections an hour by each wiremen. Those who worked above this norm suffered some degree of social ostracism. They were given nicknames such as "Speed King" and verbally chastised. They were also likely to be the butter of a game known as "binging", in which one person hit another as hard as possible on the upper arm, to which the other nominally has the right to respond by hitting back. If a large number of people chose to play the binging game with the same norm violator, this amounted to significant negative sanctions. These were the most obvious outward manifestations of the decrease in status and social belonging that accompanied a violation of the norms.

Moreover, collusive agreements among employees which aim to reduce their efforts collectively are not prohibited by law and they can even be enforced by mediators like the inspectors in the bank wiring room who were in a close relationship with the rest of workers and acted as supervisors. 
The employer at the top of the organization no doubt has little idea of the extent to which these collusive activities are organized and enforced. If the employer wants to ensure the incentive schemes are immune to collusion, the "worst" case of collusion where collusion is assumed to be accomplished by an enforcement mechanism should be taken into account, which leads to the following assumption:

Assumption 1. Collusive agreements are enforced by a benevolent mediator.

It is reasonable to assume collusion be enforced by a mediator. When enforcement is ensured by repeated interactions and reputation, enforceable side contracts at best depict a polar case in which reputation mechanisms work well to enforce collusion, as argued by Tirole (1992). This assumption is widely adopted in the literature of fighting collusion in mechanism design. Laffont and Martimort (2000) point out that this paradigm can be seen as a black box for the repeated interaction by which collusion emerges, which allows us to characterize the upper bound that can be achieved by the coalition. Moreover, they further argue that if any mechanisms are robust under perfect collusion, they must also be robust when collusion becomes imperfect. This methodology plays an important role in the research of collusion-proof mechanism design, as it provides a simple and neat approach in modelling collusion and thus avoids the problem of finding an extensive form for describing the collusive game between agents.

Given Assumption 1, the timing of the game can be illustrated as follows:

[Timing of the game]:

Stage 1. The principal proposes a tournament mechanism $M^{D}$;

Stage 2: Each agent decides to accept or not; if no one rejects then:

Stage 3: A mediator proposes a side contract $S$ to agents; each agent then decides to accept or not; if no one vetoes then:

Stage 4: Both agents choose efforts simultaneously;

Stage 5: Outcomes are realized and transfers are enforced.

A benevolent mediator aims to maximize the total welfare of the coalition, and the maximum is attained when both agents take low efforts. It appears that a simple anonymous side contract can induce both agents to shirk: Whoever wins the prize must pay a side payment $s=\frac{1}{2} T$ to his losing peer. This simple side contract offers full insurance to both agents and thus they have no incentive to work.

Whenever the principal has personal preference toward some subordinate, say agent $A_{1}$, a biased promotion rule will be employed in which case a favored agent is more likely to win than his peer given that both agents take the same efforts. The principal may favor the preferred agent in an explicit way in which case the identity of the favored one is commonly known, or in an implicit way such that the identity of the favorite remains secret. When favoritism is explicit, the favored agent has higher status quo utility than his peer and would claim for more stakes in collusion, in which case the aforementioned anonymous side contract might be insufficient to induce low efforts.

However, the mediator will instead propose an unanonymous side contract which grants more stakes from collusion to the favored agent. To fix ideas, consider a simple tournament mechanism $M^{D}$ with a biased promotion rule $H(b)$ which generates a pair of winning probability $\left(p_{1}(e ; b), p_{2}(e ; b)\right)$ for the agent $A_{1}$ and $A_{2}$ respectively. Based on this grand contract, the mediator can propose an unanonymous side contract $S=\left\{E,\left(s^{1}, s^{2}\right)\right\}$ where $E$ represents the collusive effort pair proposed by the side mediator and $s^{i}$ is the side transfer from the winner to the loser whenever agent $A_{i}$ wins. 
This side contract ensures the agent $A_{i}$ a payoff $T-s^{i}$ if he wins and $s^{j}$ otherwise. The agent $A_{i}$ 's expected utility, as denoted by $\widehat{U}_{i}(e),{ }^{17}$ is given by

$$
\widehat{U}_{i}(e)=p_{i}(e ; b)\left(T-s^{i}\right)+\left(1-p_{i}(e ; b)\right) s^{j}-C\left(e^{i}\right) .
$$

To induce low efforts, the side contract must ensure that each agent is better off than under the grand mechanism $M^{D}$, that is, the coalition participation constraint must be met for each agent:

$$
\left(C I R_{i}\right): \widehat{U}_{i}(\underline{e}) \geq U_{i}(\bar{e}), i=1,2,
$$

where $\widehat{U}_{i}(\underline{e})$ is the expected utility of the agent $A_{i}$ under collusion whilst $U_{i}(\bar{e})$ is his status quo utility.

The side contract will bring a gross stake $2 c$ for agents which must be shared between them. The favored agent $A_{1}$, however, would claim more stakes since he has higher status quo utility than his peer, while the unfavored one will participate only if he can benefit from collusion. Since reallocation of collusive stakes incurs no cost, it is possible to redistribute collusive stakes and make both agents better off. It is easy to check the following side contract: $s^{1}=p_{2}(\bar{e} ; b) T$ and $s^{2}=p_{1}(\bar{e} ; b) T$ makes both agents well-off under collusion.

Moreover, as effort levels are not observable, the side contract must also ensure that each agent has no incentive to deviate from the proposed effort level, which brings an additional coalition incentive compatibility constraint for agent $A_{i}$ :

$$
\left(C I C_{i}\right): \widehat{U}_{i}(\underline{e}) \geq \widehat{U}_{i}(\widehat{e})=p_{i}(\widehat{e} ; b)\left(T-s^{i}\right)+\left(1-p_{i}(\widehat{e} ; b)\right) s^{j}-c,
$$

where $\widehat{e} \equiv(1,0)$ denotes the effort pair whenever the agent $A_{i}$ deviates to the high effort unilaterally. In addition, the side payment that a loser can get must be non-negative since agents are protected by the limited liability:

$$
\left(C L L_{i}\right): s^{i} \geq 0
$$

The coalition incentive compatibility constraints for low effort levels $\left(C I C_{i}\right)$ requires that the virtual incentive power $T-s^{i}-s^{j}$, which is the difference of rewards between a winner and loser under collusion, be sufficiently low:

$$
\left(C I C_{i}\right):\left[p_{i}(\widehat{e} ; b)-p_{i}(\underline{e} ; b)\right]\left(T-s^{i}-s^{j}\right) \leq c .
$$

In particular, whenever the difference of rewards is reduced to zero, both agents are fully insured and thus have no incentive to work. It is easy to verify that the aforementioned side contract satisfies: $T-s^{i}-s^{j}=0$, and therefore can induce low effort levels.

The above analysis shows that, given any simple tournament mechanism $M^{D}$, there always exists a side contract $S$ that can induce low effort pair $\underline{e}$ and benefit the agents; implementing socially efficient efforts is therefore impossible as concluded by:

Proposition 2. It is impossible to induce socially efficient effort levels through any simple tournament mechanisms when collusive contracts are enforceable; in addition, only low effort levels can be implemented.

\footnotetext{
${ }^{17}$ Here"^^" represents a label of notation under collusion.
} 
The employer offers a high reward for the winner in the tournament to encourage employees to work hard; the high incentive power is however mitigated when agents reach an agreement to reduce the gap of rewards by side transfers, and as a result agents have no incentive to work. Introducing a biased promotion rule explicitly can bring a conflict in the distribution of collusive stakes as the favored agent would claim more stakes, however, this conflict can be easily reconciled by an asymmetric side contract which allows the favored agent to get more benefit from collusion, given that the identity of the favored one is common knowledge in the organization. This proposition shows a polar case of collusion under tournaments: the employer would expect that employees take only low efforts however large the rewards, whenever they can collude perfectly; it also implies that explicit favoritism is not effective in fighting collusion.

Ishiguro (2004) also shows that it is impossible to implement high efforts in tournaments when side contracts are enforceable. Moreover, he introduces a discriminatory incentive contract based on the identities of agents which grants the favored agent (and only the favored agent) a reward when he wins, assuming that the relative performance of agents can be verified according to the agents' identities. The unfavored agent always gets nothing whether he wins or loses and therefore has no incentive to work; on the other hand, the favored agent will not collude with the unfavored one as he can get no side payment from the unfavored one. Therefore, the principal can only induce the favored agent to work under the discriminatory contract.

However the incentive contract in his model involves a naked discrimination which can be verified by the court of law, therefore it cannot be applied in general cases. Moreover, this discriminatory mechanism is not robust in the cases whenever the assessments of relative performance are also subjective (which are more general in the real world). The principal has temptations to renege when the favored agent has better performance, since she will pay less by claiming that the winner is the unfavored one.

\section{COLLUSION AND STRATEGIC FAVORITISM}

Explicit favoritism is rarely observed in modern organizations. An employer who brings favoritism explicitly will be accused by employees, labor unions and even superiors, even if explicit favoritism is unlikely to be verified by the court of law. In addition, explicit favoritism is not an effective measure to fight collusion as we have shown in the impossibility theorem.

Employers often favor some subordinates implicitly and keep the identities of the favored employees as private information. Moreover, employers can reveal the information about favoritism strategically only to some employees, in order to bring asymmetric information among subordinates whenever collusion is a serious concern.

It is quite common in modern organizations that some subordinates keep closer relationships with superiors than others, ${ }^{18}$ and they may have some information about superiors' personal preference or even intend to influence the employers' preference. These subordinates are often called the informed agents who play an important role in the formation of informal communication channels among the

\footnotetext{
${ }^{18}$ For instance, these employees may have common interests about classical music with their employer, or enjoy drinking in the same pub with their boss after work.
} 
employers and employees, which can be utilized strategically by the employer to benefit organizations. ${ }^{19}$

\subsection{The Timing and Mechanisms}

The principal can introduce asymmetric information between collusive agents whenever she discloses the personal preference only to the informed agent who, whether being favored or not, would claim as the favored one. A dispute arises on the distribution of stakes, which destabilizes collusion. The mediator, when designing a side contract, must grant information rents to the informed agent in exchange of truth-telling; and this causes a trade-off between incentives of participation and truth-telling, which leads to a distortion of efficiency in collusion.

To fix ideas, we assume the principle has a close relationship with the agent $A_{1}$ who becomes the informed agent. In addition, the principal knows his preference over agents, while each agent only knows that he will be favored with probability $1 / 2$. The principal will disclose her preference only to the informed agent after both agents accept the contracts. ${ }^{20}$

The timing of the tournament with strategic favoritism is illustrated as follows:

[Timing of the mechanism]

Stage 1: The principal proposes a tournament contract $M^{I}$;

Stage 2: Each agent chooses to accept or not; if no one rejects then:

Stage 3: The principal reveals the identity of the favorite only to the informed agent $A_{1}$;

Stage 4: The mediator proposes a menu of side contracts $S$;

Stage 5: Each agent decides to accept or not. If no agent vetoes then:

Stage 6: The mediator asks the informed agent to report his type and then recommends a side contract from $S$ according to his report;

Stage 7: Both agents take efforts simultaneously;

Stage 8: Outcomes are realized and transfers are enforced.

Let subscripts " $f$ " and " $u$ " represent the type of favored and unfavored one respectively. The principal offers a grand contract $M^{I}=(\bar{e}, H(b), T)$ to agents where the promotion rule $H(b)$ induces a pair of winning probability $\left(p_{f}(e ; b), p_{u}(e ; b)\right)$ for the favored and unfavored agents as follows:

$$
p_{f}(e ; b)=G\left(e^{i}+b-e^{j}\right), \quad p_{u}(e ; b)=G\left(e^{i}-b-e^{j}\right) .
$$

Let $\Delta p(e ; b)=p_{f}(e ; b)-p_{u}(e ; b)$ denote the premium of winning probability for the favored type, we have

$$
\begin{aligned}
\Delta p(\bar{e} ; b) & =\Delta p(\underline{e} ; b)=G(b)-G(-b) \\
\Delta p(\widehat{e} ; b) & =\Delta p(\widetilde{e} ; b)=G(1+b)-G(1-b) .
\end{aligned}
$$

There are now two types of agents: the informed agent who owns private information and the uninformed agent who does not. The mediator can design either a pooling side contract which treats both agents as equal and thus ignores the private information of the informed agent; or a separating contract which requires the

\footnotetext{
${ }^{19}$ We exclude the possibility that the informed agent can be paid as a whistle-blower to report collusive activities, since we assume collusion be enforceable in the sense that any defection will be punished.

${ }^{20} \mathrm{We}$ exclude the possibility that the informed principal may propose unanonymous contracts to the favored and disfavored agents respectively, simply because the identities of agents are not verifiable.
} 
informed agent to tell the truth. We will consider the separating contract first and then discuss the pooling contract in Appendix A.

According to the Revelation Principle, without loss of generality, the mediator can propose a menu of contracts $S=\left\{\left(E_{f}, S_{f}\right),\left(E_{u}, S_{u}\right)\right\}$ to agents with each contract being selected based on the report of the informed agent. For instance the contract $\left(E_{f}, S_{f}\right)$ will be selected when the informed agent reports that he is the favored type where,

$E_{f}=\left(e_{f}^{1}, e_{u}^{2}\right)$ stands for the proposed effort pair which recommends the agent $A_{1}$ pick the effort $e_{f}^{1}$ as he is supposed to be the favored type and the agent $A_{2}$ take the effort $e_{u}^{2}$ as he is supposed to be the unfavored one;

$S_{f}=\left(s_{f}^{1}, s_{u}^{2}\right)$ is a pair of side payments from the winner to the loser. The informed agent (now the favored type) should pay $s_{f}^{1}$ to his losing peer whenever he wins, and he can get a side payment $s_{u}^{2}$ from the agent $A_{2}$ (now the unfavored type) in case he loses.

By analogy the contract $\left(E_{u}, S_{u}\right)$ will be chosen when the informed agent reports unfavored type. The side contract $S=\left\{\left(E_{f}, S_{f}\right),\left(E_{u}, S_{u}\right)\right\}$ then brings the following utility levels to agents with different types:

$$
\begin{aligned}
& \widehat{U}_{f}^{1}\left(e_{f}^{1}, e_{u}^{2}\right)=p_{f}\left(e_{f}^{1}, e_{u}^{2} ; b\right)\left(T-s_{f}^{1}\right)+\left[1-p_{f}\left(e_{f}^{1}, e_{u}^{2} ; b\right)\right] s_{u}^{2}-C\left(e_{f}^{1}\right), \\
& \widehat{U}_{u}^{1}\left(e_{u}^{1}, e_{f}^{2}\right)=p_{u}\left(e_{u}^{1}, e_{f}^{2} ; b\right)\left(T-s_{u}^{1}\right)+\left[1-p_{u}\left(e_{u}^{1}, e_{f}^{2} ; b\right)\right] s_{f}^{2}-C\left(e_{u}^{1}\right), \\
& \widehat{U}_{f}^{2}\left(e_{u}^{1}, e_{f}^{2}\right)=p_{f}\left(e_{u}^{1}, e_{f}^{2} ; b\right)\left(T-s_{f}^{2}\right)+\left[1-p_{f}\left(e_{u}^{1}, e_{f}^{2} ; b\right)\right] s_{u}^{1}-C\left(e_{f}^{2}\right), \\
& \widehat{U}_{u}^{2}\left(e_{f}^{1}, e_{u}^{2}\right)=p_{u}\left(e_{f}^{1}, e_{u}^{2} ; b\right)\left(T-s_{u}^{2}\right)+\left[1-p_{u}\left(e_{f}^{1}, e_{u}^{2} ; b\right)\right] s_{f}^{1}-C\left(e_{u}^{2}\right) .
\end{aligned}
$$

\subsection{The Possibility Theorem}

The side contract should bring each agent more benefits under collusion, so the following coalition participation constraints must be satisfied: For the informed agent $A_{1}$, whatever he is favored or not, he must get more utility from collusion:

$$
\begin{aligned}
& {\left[C I R_{f}^{1}\right]: \widehat{U}_{f}^{1}\left(e_{f}^{1}, e_{u}^{2}\right) \geq U_{f}(\bar{e}),} \\
& {\left[C I R_{u}^{1}\right]: \widehat{U}_{u}^{1}\left(e_{u}^{1}, e_{f}^{2}\right) \geq U_{u}(\bar{e}) ;}
\end{aligned}
$$

where $\left[C I R_{f}^{1}\right]\left(\left[C I R_{u}^{1}\right]\right)$ represents the coalition participation constraint when the informed agent is favored (unfavored).

Meanwhile, as the uninformed agent $A_{2}$ knows only a probability distribution over his types, he will accept the side contract if his expected utility under collusion is higher than status quo utility:

$$
\left[C I R^{2}\right]: \frac{1}{2}\left[\widehat{U}_{f}^{2}\left(e_{u}^{1}, e_{f}^{2}\right)+\widehat{U}_{u}^{2}\left(e_{f}^{1}, e_{u}^{2}\right)\right] \geq \frac{1}{2}\left[U_{f}(\bar{e})+U_{u}(\bar{e})\right] .
$$

The gross welfare of agents are fixed under the side contract since side transfers incurs no cost, which equals to the prize $T$ minus the total effort costs:

$$
\begin{aligned}
\widehat{U}^{1}+\widehat{U}^{2} & \equiv \frac{1}{2}\left[\widehat{U}_{f}^{1}\left(e_{f}^{1}, e_{u}^{2}\right)+\widehat{U}_{u}^{1}\left(e_{u}^{1}, e_{f}^{2}\right)\right]+\frac{1}{2}\left[\widehat{U}_{f}^{2}\left(e_{u}^{1}, e_{f}^{2}\right)+\widehat{U}_{u}^{2}\left(e_{f}^{1}, e_{u}^{2}\right)\right] \\
& =T-\frac{1}{2}\left[C\left(e_{f}^{1}\right)+C\left(e_{u}^{1}\right)+C\left(e_{f}^{2}\right)+C\left(e_{u}^{2}\right)\right]
\end{aligned}
$$


Let $\Psi=\frac{1}{2}\left[C\left(e_{f}^{1}\right)+C\left(e_{u}^{1}\right)+C\left(e_{f}^{2}\right)+C\left(e_{u}^{2}\right)\right]$ denote the total effort costs under collusion; we can rewrite the coalition participation constraint of the agent $A_{2}$ as follows:

$$
\left[C I R^{2}\right]: \frac{1}{2}\left[\widehat{U}_{f}^{1}\left(e_{f}^{1}, e_{u}^{2}\right)+\widehat{U}_{u}^{1}\left(e_{u}^{1}, e_{f}^{2}\right)\right] \leq T-\frac{1}{2}\left[U_{f}(\bar{e})+U_{u}(\bar{e})\right]-\Psi .
$$

Since the favored agent has higher status quo utility than his peer: $U_{f}(\bar{e})>U_{u}(\bar{e})$, the side contract must ensure the favored type more benefit under collusion. Let $\Delta \widehat{U}=\widehat{U}_{f}^{1}\left(e_{f}^{1}, e_{u}^{2}\right)-\widehat{U}_{u}^{1}\left(e_{u}^{1}, e_{f}^{2}\right)$ denote the utility premium for the favored type, combining $\left[C I R_{f}^{1}\right]$ and $\left[C I R^{2}\right]$ yields

$$
\Delta \widehat{U} \geq \Delta p(\bar{e} ; b) T-2(2 c-\Psi) .
$$

The favored type should be entitled extra utilities $\Delta \widehat{U}$ in the coalition, which is greater than the difference between the utility premium in the grand contract $\Delta p(\bar{e} ; b) T$ and the expected effort cost $2(2 c-\Psi)$.

Moreover, the side contract must be incentive compatible for the truth-telling of the informed agent. Suppose the informed one happens to be the unfavored type, then side contract $\left(E_{u}, S_{u}\right)$ will be recommended if the informed agent reports his true type. This side contract specifies an effort level $e_{u}^{1}$ (resp. $\left.e_{f}^{2}\right)$ to the informed agent $A_{1}$ (resp. the uninformed agent $A_{2}$ ) and a side payment $s_{u}^{1}\left(\operatorname{resp} . s_{f}^{2}\right)$ that the agent $A_{1}$ (resp. $\left.A_{2}\right)$ must pay his peer when he wins; it brings an expected payoff $\widehat{U}_{u}^{1}\left(e_{u}^{1}, e_{f}^{2}\right)$ to the informed agent when telling the truth:

$$
\left.\widehat{U}_{u}^{1}\left(e_{u}^{1}, e_{f}^{2}\right)=p_{u}\left(e_{u}^{1}, e_{f}^{2}\right)\left(T-s_{u}^{1}\right)+\left[1-p_{u}\left(e_{u}^{1}, e_{f}^{2}\right)\right)\right] s_{f}^{2}-C\left(e_{u}^{1}\right) .
$$

If instead the informed agent misreports his type as favored one, then the side contract $\left(E_{f}, S_{f}\right)$ is selected by analogy, which brings him an expected payoff

$$
\widehat{U}_{u, f}^{1}\left(e_{f}^{1}, e_{u}^{2}\right)=p_{u}\left(e_{f}^{1}, e_{u}^{2}\right)\left(T-s_{f}^{1}\right)+\left[1-p_{u}\left(e_{f}^{1}, e_{u}^{2}\right)\right] s_{u}^{2}-C\left(e_{f}^{1}\right),
$$

where the subscript " $u, f$ " represents that an unfavored type mimics a favored type. This payoff can be decomposed into two parts:

$$
\widehat{U}_{u, f}^{1}\left(e_{f}^{1}, e_{u}^{2}\right)=\widehat{U}_{f}^{1}\left(e_{f}^{1}, e_{u}^{2}\right)-\Delta p\left(e_{f}^{1}, e_{u}^{2}\right) T_{f}^{1},
$$

where $T_{f}^{1}=T-s_{f}^{1}-s_{u}^{2}$ represents the virtual incentive power of the favored type which is the difference of rewards between winning and losing. The unfavored type can get the expected utility of the favored type $\widehat{U}_{f}^{1}\left(e_{f}^{1}, e_{u}^{2}\right)$ by misreporting, but has to bear an information cost $\Delta p\left(e_{f}^{1}, e_{u}^{2}\right) T_{f}^{1}$ as his winning probability is $p_{u}\left(e_{f}^{1}, e_{u}^{2}\right)$ rather than $p_{f}\left(e_{f}^{1}, e_{u}^{2}\right)$. Decreasing the side payments that a favored type must pay if he wins $s_{f}^{1}$ and/or he can get if he loses $s_{u}^{2}$ would increase the virtual incentive power of the favored type, which makes it more costly for the unfavored type to mimic the favored one.

The side contract must ensure that the unfavored type cannot benefit from misreporting:

$$
\left[C T T_{u}^{1}\right]: \widehat{U}_{u}^{1}\left(e_{u}^{1}, e_{f}^{2}\right) \geq \widehat{U}_{u, f}^{1}\left(e_{f}^{1}, e_{u}^{2}\right)=\widehat{U}_{f}^{1}\left(e_{f}^{1}, e_{u}^{2}\right)-\Delta p\left(e_{f}^{1}, e_{u}^{2}\right) T_{f}^{1},
$$

or equivalently:

$$
\left[C T T_{u}^{1}\right]: \Delta \widehat{U} \leq \Delta p\left(e_{f}^{1}, e_{u}^{2}\right) T_{f}^{1}
$$


Since the unfavored type would have stronger incentives to mimic the favored one whenever the utility premium of the favored type increases, the utility premium should be reduced to mitigate this temptation of misreporting. But doing so would mitigate the incentive of the favored type to participate into collusion, as he is ensured a utility premium under the grand contract at least $\Delta p(\bar{e}) T-2(2 c-\Psi)$ as illustrated in (8). The trade-off between the incentives of participation and truth-telling can be settled only if

$$
\Delta p(\bar{e}) T-2(2 c-\Psi) \leq \Delta \widehat{U} \leq \Delta p\left(e_{f}^{1}, e_{u}^{2}\right) T_{f}^{1},
$$

and a necessary condition for this solution is that the virtual incentive power of the favored type should be large enough:

$$
T_{f}^{1} \geq \frac{\Delta p(\bar{e}) T}{\Delta p\left(e_{f}^{1}, e_{u}^{2}\right)}-\frac{2(2 c-\Psi)}{\Delta p\left(e_{f}^{1}, e_{u}^{2}\right)} .
$$

However, increasing the virtual incentive power of the favored type would bring him more incentives to deviate from the side contract and instead take the high effort which can increase his winning probability. Preventing the favored type from deviating in collusive efforts brings an additional coalition incentive compatibility constraint:

$$
\left[C I C_{f}^{1}\right]: \widehat{U}_{f}^{1}\left(e_{f}^{1}, e_{u}^{2}\right) \geq \widehat{U}_{f}^{1}\left(\widehat{e}_{f}^{1}, e_{u}^{2}\right),
$$

which ensures that the favored type cannot benefit from deviation by taking the effort $\widehat{e}_{f}^{1}$ rather than $e_{f}^{1}$ unilaterally. This requires that the virtual incentive power should be bounded above:

$$
\left[C I C_{f}^{1}\right]: T_{f}^{1} \leq \frac{C\left(\hat{e}_{f}^{1}\right)-C\left(e_{f}^{1}\right)}{\left[p_{f}\left(\widehat{e}_{f}^{1}, e_{u}^{2}\right)-p_{f}\left(e_{f}^{1}, e_{u}^{2}\right)\right]}
$$

It appears that (13) and (14) will be in contradiction when the tournament prize $T$ is large enough. To see this, suppose the side contract proposes an effort pair $E_{f}=\underline{e}=(0,0)$ for agents; we can rewrite these constraints as follows:

$$
\begin{aligned}
T_{f}^{1} & \geq \frac{\Delta p(\bar{e}) T}{\Delta p(\underline{e})}-\frac{4 c}{\Delta p(\underline{e})}, \\
T_{f}^{1} & \leq \frac{c}{\left[p_{f}(\hat{e})-p_{f}(\underline{e})\right]},
\end{aligned}
$$

where $\widehat{e} \equiv(1,0)$ represents the effort pair when the favored agent deviates to the high effort. It is obvious that these constraints contradict to each other whenever

$$
\begin{aligned}
T & \geq \widehat{T}(b) \equiv \frac{c}{\left[p_{f}(\widehat{e})-p_{f}(\underline{e})\right]}+\frac{4 c}{\Delta p(\bar{e})} \\
& =\frac{c}{[G(1+b)-G(b)]}+\frac{4 c}{[G(b)-G(-b)]},
\end{aligned}
$$

which implies there is no incentive feasible side contract $\left(E_{f}, S_{f}\right)$ implementing $E_{f}=\underline{e}$ given that the tournament prize is sufficiently high.

The mediator would instead propose a side contract to induce an asymmetric effort pair which recommends the favored type to work and the unfavored type to shirk; this is, however, also impossible when the tournament prize is high enough 
as shown by analogy (we leave this in Appendix A). Therefore, only the efficient effort pair $\bar{e}$ can be implemented in the side contract $\left(E_{f}, S_{f}\right)$.

On the other hand, if the informed agent is unfavored and reports the truth, the side contract $\left(E_{u}, S_{u}\right)$ is then recommended to the agents. In this case, the low effort pair $E_{u}=\underline{e}$ can be implemented. To see this, consider a side contract with side transfers $\left(s_{u}^{1}, s_{f}^{2}\right)$ satisfying $T_{u}^{1}=0$ such that both agents are fully insured. Both agents have no incentive to work under this side contract and therefore the coalition incentive compatibility constraints $\left[C I C_{u}^{1}\right]$ and $\left[C I C_{f}^{2}\right]$ are irrelevant. In addition, reducing the virtual incentive power $T_{u}^{1}=0$ would also prevent the favored type from mimicking the unfavored type as the information rent is diminished, which can be illustrated by analogy:

$$
\left[C T T_{l}^{1}(E)\right]: \Delta \widehat{U} \geq \Delta p\left(e_{u}^{1}, e_{l}^{2}\right) T_{u}^{1}
$$

and the trade-off between the participation incentive and the truth-telling incentive will not arise in this case. As a result, the low effort pair $\underline{e}$ can be implemented in the side contract $\left(E_{u}, S_{u}\right)$.

To sum up, the incentive feasible side contract involves $E_{f}=\bar{e}$ and $E_{u}=\underline{e}$ which recommends the high effort pair when the informed agent reports the favored type and the low effort pair otherwise. Under this contract, the socially efficient effort pair can be implemented with probability $1 / 2$ which is the likelihood that the informed agent will be favored:

Proposition 3. The socially efficient effort levels can be implemented with probability $1 / 2$ under a tournament mechanism with strategic favoritism.

Proof. See Appendix A.

Favoritism brings a utility premium in the tournament for the favored agent who should be granted more stakes in collusion. This provides incentives for the informed agent to claim as the favored type in case he happens to be unfavored. The virtual incentive power of the favored agent $T_{f}^{1}$ should be increased to mitigate this temptation of misreporting, but doing so would give the favored agent stronger incentives to take the high effort. This trade-off cannot be settled whenever the tournament prize is sufficiently high, and as a result only the high effort levels can be induced in an incentive feasible side contract $\left(E_{f}, S_{f}\right)$.

On the contrary, the favored type has no incentives to mimic the unfavored one; it is therefore feasible to reduce the virtual incentive power of the unfavored agent in a side contract $\left(E_{u}, S_{u}\right)$ which mitigates the incentives of taking high effort. In particular, side transfers $s_{u}^{1}=p_{f}(\bar{e}) T$ and $s_{f}^{2}=p_{u}(\bar{e}) T$ which satisfy $T_{u}^{1}=0$ and provide full insurance to both agents can implement the low effort pair $E_{u}=\underline{e}$.

The side contract that involves $E_{f}=\bar{e}$ and $E_{u}=\underline{e}$ reflects the classical trade-off between rent-extraction and efficiency-distortion in side-contracting. ${ }^{21}$ To attract the favored type into the coalition, the side contract must give him a high utility premium as incentive rents, which in turn provides high temptations of misreporting by the unfavored type. This calls for an increase of the virtual incentive power of the favored type to mitigate this temptation, but doing so encourages the favored type to exert the high effort. The mediator has to propose the high effort pair

\footnotetext{
${ }^{21}$ For readers not familiar with contract theory, please resort to the classical text book by Laffont and Martimort (2002). Indeed, when designing the side contract, the mediator has to deal with incentive problems that comprise adverse selection, moral hazard and countervailing incentives.
} 
$E_{f}=\bar{e}$ when the informed agent reports the favored type, and the efficiency of collusion is distorted. Although it is still possible to implement $E_{u}=\underline{e}$ when the informed agent reports the unfavored type, the stakes of collusion are reduced from $2 c$ to $c$

However, fighting collusion is costly as $\widehat{T}>T^{*}$. It appears that increasing bias $b$ can reduce the prize $\widehat{T}$ and thus benefit the principle; but doing so will reduce the likelihood of winning for the unfavored agent which in turn calls for an increase of the reward to induce high effort, as characterized by his participation constraint

$$
p_{u}(\bar{e} ; b) T \geq c,
$$

which implies

$$
T \geq T^{0}(b) \equiv \frac{c}{G(-b)} .
$$

Therefore, the optimal bias is determined when $\widehat{T}(b)=T^{0}(b)$, as given by

$$
\frac{c}{G(-b)}=\frac{c}{[G(1+b)-G(b)]}+\frac{4 c}{[G(b)-G(-b)]} .
$$

COROLLARY 1. Increasing bias can reduce the optimal reward for implementing socially efficient efforts but will increase the incentive cost for the unfavored agent; and this trade-off determines the optimal bias as illustrated by (16).

Proposition 1 shows that favoritism cannot benefit organizations where collusion is not a serious concern, in addition proposition 2 implies that explicit favoritism is not effective in dealing with well-organized collusion; while the possibility theorem of Proposition 3 shows that using favoritism strategically is an effective trick to fight collusion. Combining these results can explain why strategic favoritism prevails in organizations. There may be other reasons for the existence of favoritism in organizations, as discussed by Prendergast and Topel (1996) and Kwon (2006), whereas our analysis relates favoritism to the phenomenon of collusion and shows that strategic use of favoritism can benefit organizations whenever collusion is a serious concern.

\section{CONCLUSIONS}

Fighting collusion has long been a challenge in organizations, however it has been by and large ignored by economists with a few exceptions of the Chicago School's approach to regulatory capture in the 1970s. Since the 1980s, there has been a growing interest in studying collusive behavior, in various environments such as industrial organization, regulation and political economy, but only a few focuses on collusion inside organizations.

On the other hand, favoritism in organizations has long been attacked as one of the most important sources of workplace conflicts and stress; some even argue that perceived favoritism is a cancer within organizations. ${ }^{22}$ In spite of the widespread and significant consequence of favoritism, there are quite few economic studies on favoritism in organizations.

This paper links the phenomena of favoritism and collusion together, which seem to be irrelevant at the first glance. While most studies of favoritism focus

\footnotetext{
${ }^{22}$ From "How to Improve your Bottom Line" in the Alpha Review by Burke Group Minnesota, Inc.
} 
on its harmful effects on organizations, our analysis shows that strategic favoritism can improve the efficiency of organizations where collusion is a serious concern.

On the other hand, favoritism which brings inequality in organizations will also hurt the cooperative relationship among employees and undermine the efficiency of coordination whenever outputs depend on team rather than individual works. A trade-off between fighting collusion and promoting cooperation will arise in this case, which calls for a cautious use of strategic favoritism in organizations. We leave this study as a future task.

Our analysis on strategic favoritism also highlights the phenomenon of sophisticated personal relations between superiors and subordinates in organizations, which act as informal mechanisms complementary to formal contracts. Influence activities such as lobbying, sabotage and collusion constitute an important component of employees' daily lives, while strategic use of governance tricks like favoritism is an important skill for employers which complements incentive schemes. Economists can hardly understand the essence and consequence of incentives in organizations without knowing the interactions between these formal and informal mechanisms, therefore studies on the internal relationships inside organizations should be greatly emphasized in the future research of organization theory.

\section{Appendix A: Proof of Proposition 3}

A separating side contract $S=\left\{\left(E_{f}, S_{f}\right),\left(E_{u}, S_{u}\right)\right\}$ is incentive feasible if it satisfies all the following constraints:

The coalition participation constraints of the informed agent:

$$
\begin{array}{ll}
{\left[C I R_{f}^{1}\right]} & : \quad \widehat{U}_{f}^{1}\left(e_{f}^{1}, e_{u}^{2}\right) \geq U_{f}(\bar{e}), \\
{\left[C I R_{u}^{1}\right]} & : \quad \widehat{U}_{u}^{1}\left(e_{u}^{1}, e_{f}^{2}\right) \geq U_{u}(\bar{e}) .
\end{array}
$$

The coalition participation constraint of the uninformed agent:

$$
\left[C I R^{2}\right]: \frac{1}{2}\left[\widehat{U}_{f}^{1}\left(e_{f}^{1}, e_{u}^{2}\right)+\widehat{U}_{u}^{1}\left(e_{u}^{1}, e_{f}^{2}\right)\right] \leq T-\frac{1}{2}\left[U_{f}(\bar{e})+U_{u}(\bar{e})\right]-\Psi
$$

The coalition truth-telling constraints of the informed agent:

$$
\begin{aligned}
& {\left[C T T_{f}^{1}\right]: \widehat{U}_{f}^{1}\left(e_{f}^{1}, e_{u}^{2}\right) \geq \widehat{U}_{u}^{1}\left(e_{u}^{1}, e_{f}^{2}\right)+\left[p_{f}\left(e_{u}^{1}, e_{f}^{2}\right)-p_{u}\left(e_{u}^{1}, e_{f}^{2}\right)\right] T_{u}^{1},} \\
& {\left[C T T_{u}^{1}\right]: \widehat{U}_{u}^{1}\left(e_{u}^{1}, e_{f}^{2}\right) \geq \widehat{U}_{f}^{1}\left(e_{f}^{1}, e_{u}^{2}\right)-\left[p_{f}\left(e_{f}^{1}, e_{u}^{2}\right)-p_{u}\left(e_{f}^{1}, e_{u}^{2}\right)\right] T_{f}^{1} .}
\end{aligned}
$$

The coalition incentive compatibility constraints of the informed agent:

$$
\begin{aligned}
& {\left[C I C_{f}^{1}\right]:\left[p_{f}\left(\hat{e}_{f}^{1}, e_{u}^{2}\right)-p_{f}\left(e_{f}^{1}, e_{u}^{2}\right)\right] T_{f}^{1} \leq C\left(\hat{e}_{f}^{1}\right)-C\left(e_{f}^{1}\right),} \\
& {\left[C I C_{u}^{1}\right]:\left[p_{u}\left(\hat{e}_{u}^{1}, e_{f}^{2}\right)-p_{u}\left(e_{u}^{1}, e_{f}^{2}\right)\right] T_{u}^{1} \leq C\left(\hat{e}_{u}^{1}\right)-C\left(e_{u}^{1}\right) .}
\end{aligned}
$$

The coalition incentive compatibility constraints of the uninformed agent: ${ }^{23}$

$$
\begin{aligned}
& {\left[C I C_{f}^{2}\right]:\left[p_{f}\left(\widehat{e}_{f}^{2}, e_{u}^{1}\right)-p_{f}\left(e_{f}^{2}, e_{u}^{1}\right)\right] T_{u}^{1} \leq C\left(\widehat{e}_{f}^{2}\right)-C\left(e_{f}^{2}\right),} \\
& {\left[C I C_{u}^{2}\right]:\left[p_{u}\left(\widehat{e}_{u}^{2}, e_{f}^{1}\right)-p_{u}\left(e_{u}^{2}, e_{f}^{1}\right)\right] T_{f}^{1} \leq C\left(\widehat{e}_{u}^{2}\right)-C\left(e_{u}^{2}\right) .}
\end{aligned}
$$

\footnotetext{
${ }^{23}$ Note that the uninformed agent takes an effort following the acceptance of the side contract which reveals the information about his types.
} 
And the coalition limited liability constraints:

$$
[C L L]: s_{f}^{1}, s_{u}^{1}, s_{f}^{2}, s_{u}^{2} \geq 0 .
$$

One may argue that the side contract should also prevent the informed agent from misreporting his type and then undercutting his effort level. Indeed, this constraint is implied by the coalition truth-telling constraint which ensures that the informed agent has no incentive to lie, and the coalition incentive compatibility constraint which ensures that the informed agent has no incentive to misbehave, as one can check easily.

Consider now the agents' participation constraints. From $\left[C I R_{f}^{1}\right]$ and $\left[C I R^{2}\right]$ we get

$$
\Delta \widehat{U} \geq \Delta p(\bar{e}) T-2(2 c-\Psi) .
$$

On the other hand, by rearranging $\left[C T T_{u}^{1}\right]$ we have

$$
\left[C T T_{u}^{1}\right]: \Delta \widehat{U} \leq \Delta p\left(e_{f}^{1}, e_{u}^{2}\right) T_{f}^{1} .
$$

Therefore, combining these two constraints implies

$$
T_{f}^{1} \geq \frac{\Delta p(\bar{e}) T}{\Delta p\left(e_{f}^{1}, e_{u}^{2}\right)}-\frac{2(2 c-\Psi)}{\Delta p\left(e_{f}^{1}, e_{u}^{2}\right)} .
$$

Suppose the side contract proposes $E_{f}=\underline{e}$ or $E_{f}=\widetilde{e}$ which assigns the favored type to shirk. From $\left[C I C_{f}^{1}\right]$ we get

$$
T_{f}^{1} \leq \frac{C\left(\hat{e}_{f}^{1}\right)-C\left(e_{f}^{1}\right)}{\left[p_{f}\left(\widehat{e}_{f}^{1}, e_{u}^{2}\right)-p_{f}\left(e_{f}^{1}, e_{u}^{2}\right)\right]} .
$$

However, the constraints (13) and (14) will contradict each other when

$$
T \geq \widehat{T} \equiv \frac{C\left(\widehat{e}_{f}^{1}\right)-C\left(e_{f}^{1}\right)}{\left[p_{f}\left(\widehat{e}_{f}^{1}, e_{u}^{2}\right)-p_{f}\left(e_{f}^{1}, e_{u}^{2}\right)\right]}+\frac{2(2 c-\Psi)}{\Delta p(\bar{e})},
$$

which implies that neither $E_{f}=\underline{e}$ nor $E_{f}=\widetilde{e}$ can be implemented.

If instead the side contract proposes $E_{f}=\widehat{e}$ that assigns the favored type to work, then equation (13) will contradict the limited liability constraint $T_{f}^{1} \leq T$ when

$$
T \geq \widetilde{T} \equiv \frac{2(2 c-\Psi)}{[\Delta p(\bar{e})-\Delta p(\widehat{e})]},
$$

where $\Delta p(\bar{e} ; b)=G(b)-G(-b)$ and $\Delta p(\widehat{e} ; b)=G(1+b)-G(1-b)$. Note that $G(b)-G(-b)=2 b G^{\prime}(\zeta)$ for some $\zeta$ close to 0 and $G(1+b)-G(1-b)=2 b G^{\prime}(\eta)$ for some $\eta$ close to 1 . Since $G^{\prime}(x)$ is strictly decreasing for $x \geq 0$ and $G^{\prime}(x)=G^{\prime}(-x)$, we have $G^{\prime}(\zeta)>G^{\prime}(\eta)$ and thus $\Delta p(\bar{e} ; b)>\Delta p(\widehat{e} ; b)$. Therefore $E_{f}=\widehat{e}$ cannot be implemented too.

To sum up, there is no incentive feasible side contract $\left(E_{f}, S_{f}\right)$ that can induce any effort pairs other than the efficient one $\bar{e}$ given that

$$
T \geq \max \{\widehat{T}, \widetilde{T}\}=\widehat{T} .
$$

If the informed agent reports the unfavored type, then a side contract $\left(E_{u}, S_{u}\right)$ with payments $s_{u}^{1}=p_{f}(\bar{e}) T$ and $s_{f}^{2}=p_{u}(\bar{e}) T$ satisfying $T_{u}^{1}=0$ can implement 
the low effort pair $E_{u}=\underline{e}$, as it is easy to check that the following constraints are satisfied:

$$
\begin{gathered}
{\left[C I R_{u}^{1}\right]: \widehat{U}_{u}^{1}\left(e_{u}^{1}, e_{f}^{2}\right) \geq U_{u}(\bar{e}),} \\
{\left[C T T_{f}^{1}\right]: \widehat{U}_{f}^{1}\left(e_{f}^{1}, e_{u}^{2}\right) \geq \widehat{U}_{u}^{1}\left(e_{u}^{1}, e_{f}^{2}\right)+\left[p_{f}\left(e_{u}^{1}, e_{f}^{2}\right)-p_{u}\left(e_{u}^{1}, e_{f}^{2}\right)\right] T_{u}^{1},} \\
{\left[C T T_{u}^{1}\right]: \widehat{U}_{u}^{1}\left(e_{u}^{1}, e_{f}^{2}\right) \geq \widehat{U}_{f}^{1}\left(e_{f}^{1}, e_{u}^{2}\right)-\left[p_{f}\left(e_{f}^{1}, e_{u}^{2}\right)-p_{u}\left(e_{f}^{1}, e_{u}^{2}\right)\right] T_{f}^{1},} \\
{\left[C I C_{u}^{1}\right]:\left[p_{u}\left(\widehat{e}_{u}^{1}, e_{f}^{2}\right)-p_{u}\left(e_{u}^{1}, e_{f}^{2}\right)\right] T_{u}^{1} \leq C\left(\widehat{e}_{u}^{1}\right)-C\left(e_{u}^{1}\right),} \\
{\left[C I C_{f}^{2}\right]:\left[p_{f}\left(\widehat{e}_{f}^{2}, e_{u}^{1}\right)-p_{f}\left(e_{f}^{2}, e_{u}^{1}\right)\right] T_{u}^{1} \leq C\left(\widehat{e}_{f}^{2}\right)-C\left(e_{f}^{2}\right) .}
\end{gathered}
$$

Note that a pooling side contract is a special case of separating side contracts with $e_{f}^{1}=e_{u}^{1}=e^{1}$ and $e_{f}^{2}=e_{u}^{2}=e^{2}$, therefore no pooling contract can induce $\underline{e}$ given that the separating contracts cannot.

\section{REFERENCES}

Che, Y and J. Kim (2005) "Robustly Collusion-Proof Implementation" Econometrica 74: 1063-1107.

Chen, K (2003) "Sabotage in Promotion Tournaments" Journal of Law, Economics and Organization 19: 119-140.

Comerford, H (2002) "Findings Report Respectful Workplace Project", Government of Newfoundland and Labrador, mimeo.

Green, J and Stocky, N (1983) "A comparison of Tournament and Contracts" Journal of Political Economy 91:349-364.

Kwon, I (2006) "Endogenous Favoritism in Organizations" Topics in Theoretical Economics 6(1): 1-24.

Ishiguro, S (2004) "Collusion and Discrimination in Organizations" Journal of Economic Theory 116: 357-369.

Itoh, H (1993) "Coalitions, Incentives and Risk Sharing" Journal of Economic Theory 60: 410-427.

Kim, S, C. Qin and Y. Yu (2006) "Bribery in Rank-Order Tournaments" working paper.

Laffont, J-J and Martimort, D (1997) "Collusion under Asymmetric Information"

Econometrica, 65: 875-911.

Laffont, J-J and Martimort, D (2000) "Mechanism Design with Collusion and Correlation"

Econometrica, 68: 309-342.

Laffont, J-J and Martimort, D (2002) The Theory of Incentives 1: the PrincipalAgent Model

Princeton University Press.

Lazear, E and S. Rosen (1981) "Rank Order Tournaments as Optimal Labor Contracts"

Journal Political Economy 89:841-864.

Miller, G (1992) Managerial Dilemma: The Political Economy of Hierarchy,

Cambridge University Press, New York, NY.

Nalebuff, B and J.E. Stigilitz (1983) "Prizes and Incentives: Towards a General Theory of Compensation and Competition"

Bell Journal of Economics 13: 21-43. 
Prendergast, C (1999) "The Provision of Incentives in Firms" Journal of Economic Literature 37: 7-63.

Prendergast, C and R. Topel (1993) "Discretion and Bias in Performance Evaluation" European Economic Review 37: 355-365.

Prendergast, C and R. Topel (1996) "Favoritism in Organizations" Journal of Political Economy 104: 958-978.

Tirole, J (1986) "Hierarchies and Bureaucracies: On the Role of Collusion in Organizations"

Journal of Law, Economics and Organization, 2: 181-214.

Tirole, J (1992) "Collusion and the Theory of Organizations" In Advances in Economic Theory: Sixth World Congress, vol 2, edited by Jean-Jacques Laffont.

Varian, H (1990) "Monitoring agents with other agents" Journal of Institutional and Theoretical Economics 146: 153-174. 